\title{
Switching of edges in strongly regular graphs. I. A family of partial difference sets on 100 vertices
}

\author{
L. K. Jørgensen \\ Dept. of Math. Sciences \\ Aalborg University \\ Fr. Bajers Vej 7 \\ 9220 Aalborg, Denmark \\ leif@math.auc.dk
}

\author{
M. Klin* \\ Department of Mathematics \\ Ben-Gurion University \\ P.O.Box 653 \\ Beer-Sheva 84105, Israel. \\ klin@math.bgu.ac.il
}

Submitted: Aug 30, 2002; Accepted: Mar 29, 2003; Published: May 3, 2003

MR Subject Classifications: 05E30

\begin{abstract}
We present 15 new partial difference sets over 4 non-abelian groups of order 100 and 2 new strongly regular graphs with intransitive automorphism groups. The strongly regular graphs and corresponding partial difference sets have the following parameters: $(100,22,0,6),(100,36,14,12),(100,45,20,20),(100,44,18,20)$. The existence of strongly regular graphs with the latter set of parameters was an open question. Our method is based on combination of Galois correspondence between permutation groups and association schemes, classical Seidel's switching of edges and essential use of computer algebra packages. As a by-product, a few new amorphic association schemes with 3 classes on 100 points are discovered.
\end{abstract}

\section{Introduction}

Strongly regular graphs were frequently investigated during the last few decades in different contexts, including group theory, algebraic graph theory, design of experiments, finite geometries, error-correcting codes, etc. (see [Bro96] for a short digest of some important results in this area).

The (in a sense) most symmetric strongly regular graphs are rank 3 graphs, that is such graphs $\Gamma$ that the automorphism group $\operatorname{Aut}(\Gamma)$ acts transitively on the vertices, ordered pairs of adjacent vertices and ordered pairs of non-adjacent vertices. Rank 3 graphs play a significant role in group theory (cf. [Asc94]). The class of Cayley graphs forms a natural class of graphs with quite high symmetry: the automorphism group of a Cayley graph

\footnotetext{
*Partially supported by Department of Mathematical Sciences, University of Delaware, Newark, DE 19716
} 
acts transitively on the set of vertices, the latter can be identified with the elements of a suitable (regular) subgroup of the whole group $\operatorname{Aut}(\Gamma)$. This is why the investigation of strongly regular Cayley graphs during the last decade attracted attention of a number of experts in the area of algebraic combinatorics.

This direction is the main subject of our paper: we construct a few new strongly regular Cayley graphs, as well as we prove that certain well-known strongly regular graphs may be interpreted as Cayley graphs (all these graphs have 100 vertices).

If a Cayley graph $\Gamma=\operatorname{Cay}(H, S)$ over a group $H$ is a strongly regular graph, then the subset $S$ of $H$ (the connection set of $\Gamma$ ) is called a partial difference set in $H$. Since the pioneering paper [Ma84] by Ma it became clear that an adequate approach to the investigation of partial difference sets should be, in principle, based on the combined use of tools from permutation groups, association schemes and Schur rings. An alternative approach (which goes back to the classical theory of difference sets, see for example [Tur65]) is mostly exploiting powerful tools from number theory and character theory. It so happened that for a long time the second approach was prevailed. A lot of researchers in the area still are not aware of the many advantages of the first approach.

The main goal of our paper is to present an informal outline of a computational toolkit which enables to search quite efficiently for partial difference sets with prescribed properties. It is mainly based on the use of Galois correspondence between permutation groups and association schemes. The opportunities of developed tools are demonstrated on the discovery of a family of new partial difference sets on 100 vertices.

One more origin of our approach is the exploitation of the classical Seidel's switching of edges in strongly regular Cayley graphs. In fact systematical use and development of Seidel's ideas will hopefully be presented in the current series of papers. This first paper in the series touches the most simple and evident features of this approach.

The goal of the paper dictates its style: besides new scientific input it was our intention that it should also fulfill educational and expository loads. We were trying to bring together and to submit to a wide community of experts a number of tools (part of which may be regarded as folklore ones), which being merged together serve as a powerful computational method.

This paper consists of 9 sections. All necessary preliminaries are concentrated in Section 2. The main requested facts about switching of edges are presented in Section 3. Elementary properties of partial difference sets are discussed in Section 4.

Section 5 deals with a certain transitive permutation group $\bar{H}$ of degree 100 and order 1200 , which is a maximal subgroup of $\operatorname{Aut}\left(J_{2}\right)$. Mergings of some 2-orbits of this group leads to a number of known and new strongly regular graphs. The automorphism groups of the resulting graphs contain 4 regular non-abelian subgroups of order 100: the groups $H_{1}, H_{2}, H_{3}, H_{4}$ are introduced in Section 6. An essential part of our results is computations arranged with the aid of various computer packages (COCO, GAP, GRAPE with nauty) which are considered in section 7. A special attention is paid to COCO. This package conceptually was introduced in [FarK91] and [FarKM94], however in the current paper we use a nice option to introduce the reader to a "kitchen" of computations, including into the text a fragment of a protocol of real computations. 
Our main results are collected in Section 8; Table 8.1 contains important information about 15 new partial difference sets and 2 new strongly regular graphs with intransitive automorphism groups. The partial difference sets are explicitly presented in the same section.

A number of remarks of a historical, bibliographical, methodological and technical nature are collected in the concluding Section 9.

Besides purely computational results the paper presents also a few simple theoretical results of a possible independent interest. In a more general form some of these results (like e.g. Proposition 9) will be considered in the subsequent parts of this series.

\section{Preliminaries}

In this section we introduce some terminology from permutation group theory and algebraic combinatorics. More details may be found, for example, in [CamL91], [FarKM94], [Cam99], [Ma94] and [Asc94].

\subsection{Strongly regular graphs}

A (simple) graph $\Gamma$ consists of a finite set $V=\left\{x_{1}, \ldots, x_{v}\right\}$ of vertices and a set $E$ of 2-subsets of $V$ called edges. The adjacency matrix of $\Gamma$ (with respect to the given labelling of vertices) is a $v \times v$ matrix $A=\left(a_{i j}\right)$ such that $a_{i j}=1$ if $\left\{x_{i}, x_{j}\right\} \in E$ and $a_{i j}=0$ otherwise.

A strongly regular graph $(\mathrm{srg})$ with parameters $(v, k, \lambda, \mu)$ is a graph with $v$ vertices which is regular of valency $k$, i.e. every vertex is incident with $k$ edges, such that any pair of adjacent vertices have exactly $\lambda$ common neighbours and any pair of non-adjacent vertices have exactly $\mu$ common neighbours. An easy counting argument shows that

$$
k(k-\lambda-1)=\mu(v-k-1) .
$$

The complementary graph $\bar{\Gamma}$ of a strongly regular graph $\Gamma$ with parameters $(v, k, \lambda, \mu)$ is a strongly regular graph with parameters $(v, v-k-1, v-2 k+\mu-2, v-2 k+\lambda)$.

The adjacency matrix $A$ of a strongly regular graph satisfies the equation

$$
A^{2}=k I+\lambda A+\mu(J-I-A)
$$

where $I$ is the identity matrix and $J$ is the all ones matrix. It follows from this equation that the eigenvalues $k, r$ and $s$ of $A$ can be computed and the multiplicities $f$ and $g$ of $r$ and $s$ can be expressed in terms of the parameters $v, k, \lambda$ and $\mu$.

We say that a set $(v, k, \lambda, \mu)$ of numbers with $0 \leq k<v$ and $0 \leq \lambda, \mu \leq k$ is a feasible parameter set for a strongly regular graph if equation 1 is satisfied and the expressions $f$ and $g$ are non-negative integers.

Sometimes it is convenient to identify an edge $\{a, b\}$ of a graph with oppositely directed $\operatorname{arcs}(a, b)$ and $(b, a)$. 


\subsection{Association schemes}

A ( $d$-class) association scheme, $(X, \mathcal{R})$ consists of a finite set $X$ and a partition $\mathcal{R}=$ $\left\{R_{0}, \ldots, R_{d}\right\}$ of $X \times X$ such that

1) $R_{0}=\{(x, x) \mid x \in X\}$,

2) for each $i \in\{0, \ldots, d\}$ there exists $i^{\prime} \in\{0, \ldots, d\}$ such that

$$
R_{i^{\prime}}=\left\{(x, y) \mid(y, x) \in R_{i}\right\},
$$

3) for each triple $(i, j, k), i, j, k \in\{0, \ldots, d\}$ there exist a number $p_{i j}^{k}$ such that for all $x, y \in X$ with $(x, y) \in R_{k}$ there are exactly $p_{i j}^{k}$ elements $z \in X$ so that $(x, z) \in R_{i}$ and $(z, y) \in R_{j}$.

The numbers $p_{i j}^{k}$ are called intersection numbers.

Each $R_{i}$ may be identified with a (possibly directed) regular graph with vertex set $X$ and valency $p_{i i^{\prime}}^{0}$. We say that $p_{11^{\prime}}^{0}, \ldots, p_{d d^{\prime}}^{0}$ are the valencies of the association scheme. The association scheme is said to be primitive if each $R_{i}, i \neq 0$, is a connected graph. Otherwise we say that it is imprimitive.

An association scheme is called symmetric if $i=i^{\prime}$ for all $i \in\{0, \ldots, d\}$. If $R_{1}$ and $R_{2}$ are the relations of a symmetric 2-class association scheme then $R_{1}$ and $R_{2}$ are the edge sets of complementary strongly regular graphs. Conversely, if $R_{1}$ denotes the edge set of a strongly regular graph and $R_{2}$ is the edge set of its complement then $R_{1}$ and $R_{2}$ form a symmetric 2-class association scheme.

If $p_{i j}^{k}=p_{j i}^{k}$ for all $i, j, k \in\{0, \ldots, d\}$ then we say the association scheme it commutative. Every symmetric association scheme is commutative.

We denote the adjacency matrices of $R_{0}, \ldots, R_{d}$ by $A_{0}, \ldots, A_{d}$, respectively. If the association scheme is commutative then the matrices $A_{0}, \ldots, A_{d}$ span a $d+1$ dimensional, commutative matrix algebra called the Bose-Mesner algebra. We may generalize the above-mentioned eigenvalue computations for strongly regular graphs to get a feasibility condition for commutative association schemes.

Let $I_{0}, \ldots, I_{s}$ be a partition of $\{0, \ldots, d\}$ such that $I_{0}=\{0\}$. Then let $S_{i}=\{(x, y) \mid$ $(x, y) \in R_{j}$ for some $\left.j \in I_{i}\right\}$. Then it may happen that $\left(X,\left\{S_{0}, \ldots, S_{s}\right\}\right)$ is an association scheme. This procedure for constructing new association schemes is called merging of classes.

A symmetric association scheme $\left(X,\left\{R_{0}, \ldots, R_{d}\right\}\right)$ is called amorphic if each partition of its classes via merging produces a new association scheme. In such a case each class $R_{i}, 1 \leq i \leq d$ defines an edge set of a strongly regular graph.

A more general notion is a coherent configuration (see, e.g. [FarKM94]). However it will not be requested in our presentation. A matrix analogue of a coherent configuration usually is called a coherent algebra. 


\subsection{Permutation groups}

In this section we consider a permutation group denoted by $G$ or $(G, \Omega)$ where $\Omega$ is a finite set and $G$ is a group consisting of permutations of $\Omega$. The action of $g \in G$ on an element $x \in \Omega$ is denoted by $x^{g}$. The cardinality of $\Omega$ is called the degree of the permutation group. The orbit of an element $x \in \Omega$ is the set $\left\{x^{g} \mid g \in G\right\}$. The orbits form a partition of $\Omega$. If there is only one orbit then $(G, \Omega)$ is called transitive. If for every pair $x, y \in \Omega$ there is a unique $g \in G$ so that $x^{g}=y$ then we say that $G$ is a regular permutation group.

The stabilizer of an element $x \in \Omega$ is the subgroup $G_{x}=\left\{g \in G \mid x^{g}=x\right\}$. If $(G, \Omega)$ is a transitive permutation group and $x \in \Omega$, then the cardinalities of the orbits of $\left(G_{x}, \Omega\right)$ are called the subdegrees of $(G, \Omega)$. These are independent of the choice of $x$. The number of orbits of $\left(G_{x}, \Omega\right)$ is called the rank of $G$.

Starting from any association scheme $(X, \mathcal{R})$ we can construct a permutation group as follows. An automorphism of $(X, \mathcal{R})$ is a permutation $g$ of $X$ so that $(x, y)$ and $\left(x^{g}, y^{g}\right)$ belong to the same relation of $\mathcal{R}$ for all $x, y \in X$. The set of automorphisms form the automorphism group of $(X, \mathcal{R})$.

Conversely, we can construct an association scheme from a transitive permutation group. The permutation group $(G, \Omega)$ induces another permutation group $(G, \Omega \times \Omega)$ defined by $(x, y)^{g}=\left(x^{g}, y^{g}\right)$ for all $x, y \in \Omega$ and $g \in G$. The orbits of $(G, \Omega \times \Omega)$ are called 2-orbits of $(G, \Omega)$. The set of 2-orbits of $(G, \Omega)$ is denoted by 2-orb $(G, \Omega)$. Then 2 -orb $(G, \Omega)$ is a partition of $\Omega \times \Omega$ and if $(G, \Omega)$ is transitive then $(\Omega, 2-\operatorname{orb}(G, \Omega)$ ) is an association scheme whose valencies are the subdegrees of $(G, \Omega)$. A matrix analogue of $(\Omega, 2-\operatorname{orb}(G, \Omega))$ is called the centralizer algebra $V(G, \Omega)$ of $(G, \Omega)$. If $G$ is the full automorphism group of this association scheme then we say that $(G, \Omega)$ is 2-closed.

\subsection{Difference sets}

Let $H$ be a finite group of order $v$. Since we in particular will consider non-abelian groups, we will in most cases use multiplicative notation for $H$. The group identity in $H$ will be denoted by $e$. A $(v, k, \lambda)$ difference set in $H$ is a subset $S \subset H$ of cardinality $|S|=k$, such that each element $g \in H, g \neq e$ can be written as $g=s t^{-1}$, where $s, t \in S$, in exactly $\lambda$ ways.

If $S \subset H$ is a difference set then for any $g \in H$ the set $S g=\{s g \mid s \in S\}$ is also a difference set with the same parameters as $S$.

A difference set $S \subset H$ is used for the construction of a symmetric 2-design with the elements of $H$ as its points and the sets $S g, \quad g \in H$ as blocks. A symmetric 2-design $D$ can be constructed in this way if and only if the group $H$ is isomorphic to a group of automorphisms of $D$ acting regularly on the points. (In this case the full automorphism group $\operatorname{Aut}(D)$ of $D$ is obligatory transitive.)

\subsection{Partial difference sets}

For a group $H$ and a set $S \subset H$ with the property that $e \notin S$ and $S^{(-1)}=S$, where $S^{(-1)}=\left\{s^{-1} \mid s \in S\right\}$, the Cayley graph $\Gamma=\operatorname{Cay}(H, S)$ of $H$ with connection set $S$ is the 
graph with vertex set $H$ so that the vertices $x$ and $y$ are adjacent if and only if $x^{-1} y \in S$. Then $\Gamma$ is an undirected graph without loops.

A graph $\Gamma$ is isomorphic to a Cayley graph of a group $H$ if and only if $H$ is isomorphic to a group of automorphisms of $\Gamma$ acting regularly on the vertices. In that case the vertices of $\Gamma$ may be identified with the elements of $H$ by identifying $e$ with any vertex $x$ and $g \in H$ with $x^{g}$. Then the connection set is the set of neighbours of $e$.

For a (multiplicative) group $H$, the group ring $\mathbb{Z} H$ is the set of formal sums $\sum_{g \in H} c_{g} g$, where $c_{g} \in \mathbb{Z}$. Then $\mathbb{Z} H$ is a ring with sum

$$
\left(\sum_{g \in H} c_{g} g\right)+\left(\sum_{g \in H} d_{g} g\right)=\sum_{g \in H}\left(c_{g}+d_{g}\right) g
$$

and product

$$
\left(\sum_{g \in H} c_{g} g\right) \cdot\left(\sum_{g \in H} d_{g} g\right)=\sum_{g \in H}\left(\sum_{h \in H} c_{h} d_{h^{-1} g}\right) g .
$$

For a set $S \subseteq H$ we define $\underline{S}=\sum_{g \in S} g \in \mathbb{Z} H$. We write $\{g\}$ as $g$. The set difference of $H$ and $S$ is denoted by $H-S$ and $H-\{e\}$ is also written as $H-e$.

A subset $S \subset H$ of a group $H$ of order $v$ is a $(v, k, \lambda)$ difference set if and only if the equation $\underline{S} \cdot \underline{S^{(-1)}}=k e+\lambda \underline{H-e}$ is satisfied in the group ring.

We say that $S \subset H$ with $|S|=k$ is a partial difference set (pds) with parameters $(v, k, \lambda, \mu)$ if, in the group ring, we have

$$
\underline{S} \cdot \underline{S^{(-1)}}=\gamma e+\lambda \underline{S}+\mu \underline{H-S},
$$

for some number $\gamma$.

Any $(v, k, \lambda)$ difference set is a $(v, k, \lambda, \lambda)$ partial difference set.

A partial difference set $S$ is called reversible if $S=S^{(-1)}$. A reversible partial difference set, $S$, is called regular if $e \notin S$.

A Cayley graph $\operatorname{Cay}(H, S)$ is a strongly regular graph if and only if $S$ is a regular partial difference set.

Suppose that $S_{1}$ and $S_{2}$ are difference sets or partial difference sets in a group $H$, and suppose that there exist an automorphism of $H$ that maps $S_{1}$ to $S_{2}$. Then in a characterization of (partial) difference sets in $H, S_{1}$ and $S_{2}$ will considered to be the same (more exactly CI-equivalent, where CI stands for Cayley isomorphism, cf. [Bab77]).

We note that even if $S_{1}$ and $S_{2}$ are two different partial difference sets in $H$ (i.e., no group automorphism maps $S_{1}$ to $\left.S_{2}\right)$, it is possible that the graphs $\operatorname{Cay}\left(H, S_{1}\right)$ and $\operatorname{Cay}\left(H, S_{2}\right)$ are isomorphic.

\subsection{Sporadic simple groups}

Some of the finite simple groups are related to strongly regular graphs in the sense that they possess rank 3 actions and thus a (symmetric) 2-orbit is a strongly regular graph.

In addition to the infinite families there are 26 sporadic finite simple groups. Two of these sporadic groups have rank 3 actions on 100 points. 
The Higman-Sims group denoted by HS has order 44352000. It was first constructed as a subgroup of index 2 of the full automorphism group of the unique strongly regular graph with parameters $(100,22,0,6)$. The graph and the group were constructed by Higman and Sims [HigS68]. The uniqueness of the graph was proved by Gewirtz [Gew69]. The automorphism group of the graph is Aut(HS).

The Hall-Janko-Wales group denoted by $J_{2}$ has order 604800 . It was first constructed by Hall and Wales [HalW68] but its existence was predicted by Janko [Jan69]. The 2orbits of its rank 3 action on 100 points are strongly regular graphs with parameters $(100,36,14,12)$ and $(100,63,38,40)$. The automorphism group of these graphs is $\operatorname{Aut}\left(J_{2}\right)$. $J_{2}$ is a subgroup of $\operatorname{Aut}\left(J_{2}\right)$ of index 2 .

The strongly regular graphs of Hall-Wales with parameters $(100,36,14,12)$ and HigmanSims with parameters $(100,22,0,6)$ will be denoted by $\Theta$ and $\Xi$, respectively, in this paper.

\section{Switching of edges in srg's}

Let $\Gamma$ be any graph and let $\left\{V_{1}, V_{2}\right\}$ be a partition of the vertex set of $\Gamma$. Let $E_{1}=$ $\left\{\{u, v\} \mid u \in V_{1}, v \in V_{2},\{u, v\} \in E(\Gamma)\right\}$ and $E_{2}=\left\{\{u, v\} \mid u \in V_{1}, v \in V_{2},\{u, v\} \notin E(\Gamma)\right\}$. Then switching of edges with respect to the partition $\left\{V_{1}, V_{2}\right\}$ means to delete the edges $E_{1}$ from $\Gamma$ and to add new edges $E_{2}$, i.e. it means to switch edges and non-edges between $V_{1}$ and $V_{2}$. Switching was introduced by Seidel in [Sei67], see Section 9 for more details.

Our motivation for considering switching of edges in graphs is the fact that if certain conditions are satisfied then switching of edges in a strongly regular graph may produce another strongly regular graph.

If switching of edges in a regular graph produces a regular graph then the corresponding partition provides a particular case of the following notion.

Definition 1 A partition $\left\{V_{1}, \ldots, V_{n}\right\}$ of the vertex set of a graph is called equitable if there exist numbers $c_{i j}, i, j \in\{1, \ldots, n\}$ such that every vertex in $V_{i}$ has exactly $c_{i j}$ neighbours in $V_{j}$, for $i, j=1, \ldots, n$.

Proposition 2 The partition into vertex orbits under the action of a group of automorphisms of a graph provides an equitable partition.

Suppose that $\left\{V_{1}, V_{2}\right\}$ is an equitable partition of the vertices of a strongly regular graph into two sets with $\left|V_{1}\right|=\left|V_{2}\right|=\frac{v}{2}$. Then the number of edges between $V_{1}$ and $V_{2}$ is $\frac{v}{2} c_{12}=\frac{v}{2} c_{21}$. Write $c=c_{12}=c_{21}$. In this case we may get a strongly regular graph with new parameters by switching with respect to $\left\{V_{1}, V_{2}\right\}$.

Proposition 3 Let $\Gamma$ be a strongly regular graph with parameters $(v, k, \lambda, \mu)$ satisfying the equation $\frac{v}{2}=2 k-\lambda-\mu$. Let $\left\{V_{1}, V_{2}\right\}$ be an equitable partition of the vertices of $\Gamma$ into two sets of equal size. Then the graph obtained by switching with respect to $\left\{V_{1}, V_{2}\right\}$ is a strongly regular graph with parameters $(v, k+a, \lambda+a, \mu+a)$, where $a=\frac{v}{2}-2 c$ and $c=c_{12}=c_{21}$. 
Proof Let $\Gamma^{\prime}$ denote the graph obtained by switching edges in $\Gamma$ with respect to the partition $\left\{V_{1}, V_{2}\right\}$. By switching we delete $c$ edges incident with each vertex and add $\frac{v}{2}-c$ new edges. Thus $\Gamma^{\prime}$ is regular of degree $k+\frac{v}{2}-2 c$.

Let $x$ and $y$ be vertices in $\Gamma$ and let $d_{i}$ denote the number of common neighbours of $x$ and $y$ in $V_{i}, i=1,2$. Clearly, $d_{1}+d_{2}=\lambda$ or $\mu$ depending on whether $x$ and $y$ are adjacent or not. Suppose first that $x, y \in V_{1}$. Then, in $V_{2}, d_{2}$ vertices are adjacent to both $x$ and $y, c-d_{2}$ vertices are adjacent to $x$ but not to $y, c-d_{2}$ vertices are adjacent to $y$ but not to $x$ and thus $\frac{v}{2}-2\left(c-d_{2}\right)-d_{2}=d_{2}+\frac{v}{2}-2 c$ vertices in $V_{2}$ are not adjacent to $x$ or $y$. In $\Gamma^{\prime}, x$ and $y$ have in total $d_{1}+d_{2}+\frac{v}{2}-2 c$ common neighbours.

Similarly, if $x$ and $y$ are both in $V_{2}$ then the number of common neighbours of $x$ and $y$ is increased by $\frac{v}{2}-2 c$ after switching.

Now suppose that $x \in V_{1}$ and $y \in V_{2}$. Then, in $V_{1}, x$ has $k-c$ neighbours; $d_{1}$ of these are also neighbours of $y$. In $\Gamma^{\prime}, x$ and $y$ have $k-c-d_{1}$ common neighbours in $V_{1}$ and similarly they have $k-c-d_{2}$ common neighbours in $V_{2}$; in total $2 k-2 c-\left(d_{1}+d_{2}\right)$.

Thus the new graph is strongly regular with parameters $\left(v, k+\frac{v}{2}-2 c, \lambda^{\prime}, \mu^{\prime}\right)$ if and only if

$$
\lambda^{\prime}=\lambda+\frac{v}{2}-2 c=2 k-2 c-\mu
$$

and

$$
\mu^{\prime}=\mu+\frac{v}{2}-2 c=2 k-2 c-\lambda
$$

i.e. it is strongly regular if and only if $\frac{v}{2}=2 k-\mu-\lambda$.

Remark. Note that the formulation of Proposition 3 does not specify the value of $c$ as a function of the parameters $v, k, \lambda, \mu$. However using some other counting techniques or with the aid of the spectrum of $\Gamma$ it can be shown that $c=\frac{2 k+\mu-\lambda \pm \sqrt{(\mu-\lambda)^{2}+4 k-4 \mu}}{4}$.

Corollary 4 If $\Gamma$ is a strongly regular graph with $\frac{v}{2}=2 k-\mu-\lambda$ and if $\operatorname{Aut}(\Gamma)$ has an intransitive subgroup with exactly two orbits and these orbits have equal size then the graph obtained by switching with respect to the partition into orbits is strongly regular.

We will in particular consider the case where the automorphism group of a strongly regular graph (with $\frac{v}{2}=2 k-\mu-\lambda$ ) has a regular subgroup and this subgroup has a subgroup of index 2. We will first consider in general strongly regular graphs with a regular group of automorphisms.

\section{Elementary properties of partial difference sets}

In this section we collect a few simple propositions about partial difference sets which will be used by us in the subsequent part of this paper. We refer to Ma [Ma84] and [Ma94] for a detailed discussion of elementary properties of partial difference sets.

Proposition 5 Suppose that $D$ is a $(v, k, \lambda, \mu)$ pds in a group $H$. Then $H-D$ is a $(v, v-k, v-2 k+\mu, v-2 k+\lambda) p d s$ in $H$. 
Proof It is clear that $(H-D)^{(-1)}=H-D^{(-1)}$. Therefore from the equality $\underline{D} \cdot \underline{D^{(-1)}}=$ $\lambda \underline{D}+\mu(\underline{H}-\underline{D})+\gamma e$ it follows that $(H-D) \cdot(H-D)^{(-1)}=(\underline{H}-\underline{D}) \cdot\left(\underline{H}-\underline{D^{(-1)}}\right)=$ $\underline{H} \cdot \underline{H}-\underline{D} \cdot \underline{H}-\underline{H} \cdot \underline{D^{(-1)}}+\underline{D} \cdot \underline{D^{(-1)}}=v \underline{H}-\overline{2 k \underline{H}+\lambda D}+\mu \underline{H-D}+\gamma e=(v-2 k+$ $\lambda) \underline{D}+(v-2 k+\mu) \underline{H-D}+\gamma e=(v-2 k+\mu) \underline{H-D}+(v-2 k+\lambda)(\underline{H}-\underline{H-D})+\gamma e$.

Proposition 6 Suppose that $D$ is a reversible $(v, k, \lambda, \mu)$ pds in a group $H$, such that $e \in D$. Then $(D-e)$ is a regular $(v, k-1, \lambda-2, \mu)$ pds in $H$. Conversely, if $D$ is a regular pds in $H$ then $D+e$ is a reversible pds with corresponding parameters.

Proof According to the assumption of the proposition, we have $\underline{D} \cdot \underline{D^{(-1)}}=\underline{D} \cdot \underline{D}=$ $\lambda \underline{D}+\mu \underline{H-D}+\gamma e$. Therefore, $\underline{D-e} \cdot \underline{D-e}=\underline{D} \cdot \underline{D}-2 \underline{D}+e=\lambda \underline{D}+\mu \underline{H-D}+\gamma e-2 \underline{D}+e=$ $(\lambda-2) \underline{D}+\mu \underline{H-D}+(\gamma+1) e=(\lambda-2) \underline{D-e}+\mu \underline{H-D+e}+(\gamma+\lambda-\mu-1) e$.

Proposition 7 Suppose that $D$ is a $(v, k, \lambda)$ difference set in $H$. Then for each $x \in H$ the shift $D x$ is also a $(v, k, \lambda)$ difference set in $H$.

Proof $\underline{D x} \cdot \underline{(D x)^{(-1)}}=\underline{D} \cdot x \cdot x^{-1} \cdot \underline{D^{(-1)}}=k e+\lambda \underline{G-e}$.

Corollary 8 Suppose that $D$ is a $(v, k, \lambda)$ difference set in $H, x \in H$. Then

- Dx is a regular $(v, k, \lambda, \lambda)$ pds if and only if $x^{-1} \notin D$ and $D x$ is a reversible set,

- $D x-e$ is a regular $(v, k, \lambda-2, \lambda)$ pds if and only if $x^{-1} \in D$ and $D x$ is a reversible set.

Corollary 8 provides a simple and efficient procedure for the search of regular pds's starting from a known difference set $D$. For this purpose it is necessary:

- to construct all shifts $D x$ of $D, x \in H$,

- to select those shifts which are reversible sets in $H$,

- each shift which does not contain $e$ is a regular $(v, k, \lambda, \lambda)$ pds,

- each shift which includes $e$ implies a regular $(v, k, \lambda-2, \lambda)$ pds $D x-e$.

In what follows we will call this method the shift procedure. Note that, in principle, different shifts may produce non-equivalent pds's or even non-isomorphic srg's.

Example 1 (a) One of the simplest examples, which properly illustrates the abovedescribed procedure, can be constructed on 16 points. Following Exercise 2.10 in Hughes and Piper [HugP85], let us consider in the elementary abelian group $H=V_{4}(2)=\left(\mathbb{Z}_{2}\right)^{4}$ a subset $D_{1}=\{0000,1000,0100,0010,0001,1111\}$. It is easy to see that $D_{1}$ is a $(16,6,2)$ difference set in $H$. Since, for each $x \in H$, the inverse element of $x$ coincides with $x$, all shifts of $D_{1}$ (we use here additive notation) are reversible. Therefore we get, using shifts, 6 regular pds's with the parameters $(16,5,0,2)$ and 10 regular pds's with the parameters $(16,6,2,2)$. In particular, $D_{2}=D_{1}-0000=\{1000,0100,0010,0001,1111\}$ 
is a regular pds, which implies the well-known Clebsch graph (see Klin, Pöschel and Rosenbaum [KliPR88] for more details about srg's appearing in this example), $D_{3}=$ $\left(D_{1} \oplus 0001\right)-0001$ produces an $L_{2}(4)$. Note that the shifts of $D_{1}$ produce the "nicest" biplane $B$ (in the notation of Hughes and Piper [HugP85], see also [Rog84]) which has doubly transitive automorphism group of order $2^{4} \cdot 6$ !.

(b) Now let us consider a group $H=\left(\mathbb{Z}_{4}\right)^{2}$, and let $D_{4}=\{01,03,10,13,30,31\}$. One can easily check that $D_{4}$ is also a $(16,6,2)$ difference set. Clearly, $D_{4}$ is a regular pds. This pds defines another srg with the parameters $(16,6,2,2)$ which is well-known under the name Shrikhande graph. In this case not all shifts of $D_{4}$ lead to reversible sets, for example, $D_{4} \oplus 01$ is not reversible. However, we can get here another pds $D_{5}=D_{4} \oplus 22=\{12,13,21,23,31,32\}$ which also produces the Shrikhande graph. We refer to $[\mathrm{HeiK}]$ for a more detailed analysis of various links between pds's on 16 points.

Now we introduce one more technique for the manipulations with pds's which is based on the use of switching of edges in the corresponding srg's. It turns out that in certain cases such switching can be properly formulated in terms of the group algebra over $H$.

Proposition 9 Suppose that $D$ is a regular pds with parameters $(4 n, k, \lambda, \lambda)$ over a group $H$ of order $4 n$. Suppose there exists such subgroup $H^{\prime}$ of index 2 in $H$ that $\left|D^{\prime}\right|=n$, where $D^{\prime}=D \cap H^{\prime}$. Let $\bar{D}=D-D^{\prime} \cup\left(H^{\prime}-D^{\prime}-e\right)$. Then $\bar{D}$ is a regular $p d s$ over the same group $H$ with the parameters $(4 n, k-1, \lambda-2, \lambda)$.

Proof The proof is based on the use of propositions proved in section 3. We have to check that for the $\operatorname{srg} \Gamma=\operatorname{Cay}(H, D)$ the partition $\tau=\left\{H^{\prime}, H-H^{\prime}\right\}$ satisfies all assumptions of Proposition 3. The fact that $\tau$ is an equitable partition follows immediately from Proposition 2, see also Corollary 4. An easy counting (cf. Remark in Section 3) shows that the existence of such equitable partition implies that $k=n+\lambda$, i.e., $\frac{v}{2}=2 k-\lambda-\mu$ (and also $\lambda=n \pm \sqrt{n}$ ). The srg $\Gamma^{\prime}$ obtained by switching with respect to $\tau$ has parameters $(4 n, k+a, \lambda+a, \mu+a)$, where $a=2 n-2 \lambda$. Cay $(H, \bar{D})$ is the complement of $\Gamma^{\prime}$.

Example 2 (Continuation of Example 1). Here $v=4 n=16, n=4$. Let $H=\left(\mathbb{Z}_{2}\right)^{4}$, $D_{1}$ as was defined above. Let $D_{6}=D_{1} \oplus 0011=\{0011,1011,0111,0001,0010,1100\}$. Let us consider as $H^{\prime}$ the subgroup of $H$ which is defined by the equation $x_{1}=0$. Then the intersection $H^{\prime} \cap D_{6}$ has cardinality $n=4$, therefore all assumptions of Proposition 9 are satisfied. Therefore we get a new pds $D_{7}$ with the parameters $(16,5,0,2), D_{7}=$ $\{0100,0101,0110,1011,1100\}$.

Remark. As it was mentioned in the introduction, Proposition 9 may be formulated and proved with weaker assumptions. In this paper we restrict our attention to a particular case which is suitable for our main goal of the investigation of pds's on 100 vertices.

\section{$5 \quad$ Starting permutation group}

The starting point for our computations was the following fact (for more details, see [FinR73], [FisM78], [IvaKF82]). 
The simple group of Hall-Janko-Wales, $J_{2}$, of order 604800 has a maximal subgroup $H$ of order 600 which is isomorphic to the direct product $D_{5} \times A_{5}$, where $D_{5}$ is the dihedral group of order 10 and $A_{5}$ is the alternating group of degree 5. Group $H$ as a subgroup of the automorphism group of an $\operatorname{srg} \Theta$ with the parameters $(100,36,14,12)$ is acting transitively on 100 vertices.

We decided to construct this action and to describe all association schemes which appear as merging of classes in the scheme of 2-orbits of this action of $H$. It was clear from the beginning that one of the resulting schemes will give the graph $\Theta$, however we were hoping to get other interesting association schemes. Fortunately, this hope was indeed justified.

All computations were done with the aid of computer package COCO, see section 7 for more details.

It turns out that $H$ has rank 24 , and it is 2-closed. The association scheme of 2-orbits of $H$ has 125 non-trivial mergings, 10 of which are primitive. These primitive association schemes were the main target of our interest. On next step of computations we tried group $\bar{H}$ of order 1200 which is an overgroup of $H$. By definition, $\bar{H}$ is the normalizer of $H$ in the full automorphism group of the graph $\Theta$. This group $A u t(\Theta)$ has $J_{2}$ as a subgroup of index 2. In principle, using information about maximal subgroups together with the argumentation presented in [Wil85], one can easily describe the structure of $\bar{H}$. In order to make our presentation self-contained we prefer to give here direct description of $\bar{H}$, as it was obtained by COCO. All above information may be used by the reader as a motivation of the appearance of $\bar{H}$.

Therefore we restart with the definition of the group $\bar{H}=\left(F_{5}^{20} \times S_{5}\right)^{\text {pos }}$. At the beginning we consider this group as an intransitive group acting on the set $\{0, \ldots, 9\}$. The group $\bar{H}$ is a subgroup of index 2 in the direct product of the Frobenius group $F_{5}^{20}$ of order 20 and the symmetric group $S_{5}$. This subgroup consists of all even permutations in $F_{5}^{20} \times S_{5}$. It is clear that $\bar{H}=\left\langle g_{1}, g_{2}, g_{3}, g_{4}, g_{5}\right\rangle$, where $g_{1}=(0,1,2,3,4), g_{2}=(5,6,7,8,9)$, $g_{3}=(1,4)(2,3), g_{4}=(5,6,7)$, and $g_{5}=(1,2,4,3)(6,7,9,8)$.

Let $\alpha=(0,\{(5,6),(6,7),(7,5)\})$ be a simple combinatorial structure which consists of a point 0 and a directed triangle with the vertices $\{5,6,7\}$. One can easily check that the subgroup $K$ of $\bar{H}$ which stabilizes $\alpha$ has order 12 and is isomorphic to $\left(\mathbb{Z}_{4} \times \mathbb{Z}_{3} \times \mathbb{Z}_{2}\right)^{\text {pos }}$. More precisely, $K=\left\langle g_{3}, g_{4}, g_{6}\right\rangle$, where $g_{6}=(1,2,4,3)(8,9)$. Let us now consider the transitive faithful action of $\bar{H}$ on the set $\Omega$ of right cosets with respect to $K$. According to the main paradigm of COCO, it is convenient to identify $\Omega$ with the set $\left\{\alpha^{g} \mid g \in \bar{H}\right\}$ of all the images of the structure $\alpha$ under the initial action of $\bar{H}$. This 100 -element set was indeed constructed with the aid of the function "inducing" of COCO.

Below we collect some other results about the permutation group $(\bar{H}, \Omega)$ and results related to its association scheme $\mathcal{M}=(\Omega, 2-\operatorname{orb}(\bar{H}, \Omega))$ which were obtained with the aid of functions from COCO.

Fact 1. $(\bar{H}, \Omega)$ is a transitive permutation group of rank 13 with the subdegrees $1^{2}, 4^{2}, 6^{3}$, $12^{4+2 \times 1}$ (here, for example like in [FarKM94], $4^{2}$ means two subdegrees equal to 4 corresponding to symmetric 2 -orbits, while $12^{4+2 \times 1}$ means 6 subdegrees equal to 12 , one pair of which corresponds to antisymmetrical 2-orbits). 
Fact 2. $(\bar{H}, \Omega)$ is a 2-closed imprimitive permutation group of order 1200 .

Fact 3. The association scheme $\mathcal{M}$ allows 39 non-trivial mergings of classes, including 10 primitive mergings of classes. All these primitive mergings correspond to srg's, in particular, 2 srg's with the parameters $(100,22,0,6), 4$ srg's with the parameters $(100,36,14,12)$, and 4 srg's with the parameters $(100,45,20,20)$. There exist 4 mergings, each of which is an imprimitive amorphic association scheme with 3 classes having the valencies 36, 9, 54 .

A few further facts were obtained using besides COCO also GAP [GAP] and its share package GRAPE [Soi93], including nauty [McKay90].

Fact 4. Up to isomorphisms we get just one srg for each parameter set with the automorphism groups Aut(HS), Aut $\left(J_{2}\right)$, and $\bar{H}$ respectively, where HS and $J_{2}$ are the sporadic simple groups of Higman-Sims and Hall-Janko-Wales. All 4 amorphic association schemes are also isomorphic.

Fact 5. The above-mentioned amorphic association scheme with the valencies 36, 9, 54 may be interpreted as follows. Consider srg $\bar{\Theta}$ - the complementary graph to the above mentioned $\operatorname{srg} \Theta$. This graph has a spread which consists of 10 disjoint 10-vertex cliques. Deletion of this spread from the edge set of $\bar{\Theta}$ leads to a new srg with the parameters $(100,45,20,20)$.

Fact 6. Graph $\bar{\Theta}$ has exactly 280 different 10 -vertex cliques on which $J_{2}$ and $\operatorname{Aut}\left(J_{2}\right)$ are acting primitively as rank 4 groups. The 2 -orbits of this action form an association scheme with 3 classes, having valencies $36,108,135$. Two of three possible mergings imply strongly regular graphs on 280 vertices (these were discovered in [IvaKF82], [IvaKF84], see also [FarKM94], and independently in [Bag88]). One of these srg's $\Delta$ has valency 144. In this srg, two vertices (anticliques of $\Theta$ ) are adjacent iff they are disjoint. Therefore a spread in $\bar{\Theta}$ corresponds to a clique of size 10 in this new $\operatorname{srg} \Delta$. It turns out that $\Delta$ has four orbits of these cliques with respect to the action of $\operatorname{Aut}\left(J_{2}\right)$ having length 1008, 12096, 12096 and 12096, respectively. The representatives of these orbits may be used for the construction of two new strongly regular graphs, namely: $\Gamma_{1}$ and three isomorphic copies of a graph $\Gamma_{2}$ with the parameters $(100,45,20,20)$, and with the automorphism groups of order 1200 and 100 respectively. The switching procedure gives also four amorphic association schemes with the valencies $36,9,54$, see Corollary 13 . The graph $\Gamma_{1}$ is isomorphic to the new srg which was discussed above. The graph $\Gamma_{2}$ is one more new strongly regular graph obtained by us. We will discuss additional properties of these graphs in the following sections.

Remark. In principle, the existence of such spreads in $\bar{\Theta}$ can also be deduced from the information about intersections of maximal subgroups in $J_{2}$ which is presented in [KomT86].

\section{Groups of order 100.}

A part of the main results of this paper consists of the proof of the existence of partial difference sets over four groups of order 100. All these groups are non-abelian. These groups will be introduced below. 
$H_{1}$ is the group generated by $x, y, z$ with relations $x^{5}=y^{5}=z^{4}=e, x y=y x, z x=$ $x^{3} z, z y=y z$.

$H_{2}$ is the group generated by $x, y, z$ with relations $x^{5}=y^{5}=z^{4}=e, x y=y x, z x=$ $x^{3} z, z y=y^{4} z$.

$H_{3}$ is the group generated by $x, y, z$ with relations $x^{5}=y^{5}=z^{4}=e, x y=y x, z x=$ $x^{2} z, z y=y^{2} z$.

$H_{4}$ is the group generated by $x, y, z$ with relations $x^{5}=y^{5}=z^{4}=e, x y=y x, z x=$ $x^{2} z, z y=y^{3} z$.

In the GAP catalogue of groups of order $100, H_{1}, H_{2}, H_{3}$, and $H_{4}$ have numbers 9 , 10,11 , and 12 , respectively. In principle the above relations are sufficient to identify each group. However, it turns out that all four groups can be represented in a similar manner as intransitive permutation groups of degree 10. Extending notation introduced in section 5 , let us put $g_{1}=(0,1,2,3,4), g_{2}=(5,6,7,8,9), g_{5}=(1,2,4,3)(6,7,9,8), g_{7}=(1,2,4,3)$, $g_{8}=(1,2,4,3)(6,9)(8,7), g_{9}=(1,3,4,2)(6,7,9,8)$.

Then for all four groups we assign $x=g_{1}, y=g_{2}$, while $z=g_{7}$ for $H_{1}, z=g_{8}$ for $H_{2}$, $z=g_{5}^{-1}$ for $H_{3}, z=g_{9}$ for $H_{4}$.

An additional advantage of this representation is that the desired actions of the groups $H_{3}$ and $H_{4}$ may be proceeded in a similar manner as for the group $\bar{H}$ in section 5: we have to take induced action of these groups on the set of all images of the combinatorial structure $\alpha=(0,\{(5,6),(6,7),(7,5)\})$. In each case we get a transitive induced permutation group of degree 100. In principle, this information is enough for further presentation of the new partial difference sets. However, we were able to prove that all regular subgroups of the groups $\operatorname{Aut}\left(J_{2}\right)$ and $\operatorname{Aut}(H S)$ are contained in the above list. We think that this fact is of an independent interest for the reader. This is why the formulations of corresponding propositions and outlines of their proofs are given below.

Proposition 10 The group $J_{2}$ in its action of degree 100 does not have any regular subgroup. The automorphism group Aut $\left(J_{2}\right)$ in its action of degree 100 has exactly two classes of conjugate regular subgroups of order 100. The representatives of these classes are the groups $\mathrm{H}_{3}$ and $\mathrm{H}_{4}$.

Proof It was shown above that $H_{3}$ and $H_{4}$ are indeed regular subgroups of $\bar{H}$ (and therefore of $\left.\operatorname{Aut}\left(J_{2}\right)\right)$. Recall that $\left|J_{2}\right|=604800$ and $\left|\operatorname{Aut}\left(J_{2}\right)\right|=2\left|J_{2}\right|$. Therefore a Sylow 5 -subgroup of these groups has order 25 .

Let $K$ be a regular subgroup of Aut $\left(J_{2}\right)$. Then a Sylow 5-subgroup of $K$ also has order 25. Let us fix a Sylow 5 -subgroup $S$ of $\operatorname{Aut}\left(J_{2}\right)$ and let us consider only those groups $K$ which contain $S$.

By Sylow's theorems, $S$ is a normal subgroup of $K$. Therefore $K$ is a subgroup of the normalizer of $K$ in $\operatorname{Aut}\left(J_{2}\right)$. This normalizer $N$ can be computed in GAP. It is a group of order 600 with 55 elements of order 2 and 200 elements of order 4 . (In fact, $N$ is a maximal subgroup of Aut $\left(J_{2}\right)$, see for example, [IvaKF84], [FarKM94].)

Routine inspection in GAP shows that $N$ has no subgroup of order 100 generated by $S$ and (at most) two elements of order 2 and that each subgroup of $N$ generated by $S$ and an element of order 4 is a regular subgroup. 
In total, $N$ has four regular subgroups: one regular subgroup isomorphic to $H_{3}$ and three regular subgroups isomorphic to $H_{4}$. Computation in GAP shows that the regular subgroups of $N$ isomorphic to $H_{4}$ are conjugate in $N$.

Thus there are just two conjugacy classes of regular subgroups in $\operatorname{Aut}\left(J_{2}\right)$. None of these groups are subgroups of $J_{2}$. In fact the normalizer of $S$ in $J_{2}$ has order 300 and is a maximal subgroup of $J_{2}$, but it has no elements of order 4 .

Proposition 11 The group HS in its action of degree 100 does not have any regular subgroups. The automorphism group Aut(HS) in its action of degree 100 has exactly four classes of conjugate subgroups of order 100. The representatives of these classes are the groups $H_{1}, H_{2}, H_{3}, H_{4}$.

Proof In this case our proof requires more computations in GAP. We give below just its short sketch, omitting some technical details of computation in GAP.

- Find a Sylow 5-subgroup S of Aut(HS) of order 125.

- $S$ has six subgroups of order 25, four of them are not semiregular (in the action on 100 points). The other two are conjugate in Aut(HS). Let $L$ be one of them.

- Again $L$ is a normal subgroup of a prospective regular subgroup of order 100. Therefore we may consider only those subgroups which are contained in the normalizer $N$ of $L$ in Aut(HS). $N$ has order 2000. It has 75 elements of order 2 and 900 elements of order 4 .

- Routine inspection shows that all subgroups of $N$ generated by $L$ and two elements of order 2 are not regular, while there are 16 regular subgroups of $N$ which are generated by $L$ and one of the elements of order 4 .

- The above 16 regular subgroups belong to four different conjugacy classes of Aut(HS). The representatives of these are the groups $\mathrm{H}_{1}, \mathrm{H}_{2}, \mathrm{H}_{3}, \mathrm{H}_{4}$.

- None of the subgroups $H_{1}, H_{2}, H_{3}, H_{4}$ are subgroups of HS, i.e., HS does not have any regular subgroup.

This completes the proof.

Remark. All maximal subgroups of HS are known ([Mag71]). Taking this information into account, the group $L$ can be identified as a subgroup of the stabilizer in Aut(HS) of the partition of the Higman-Sims graph into two copies of the Hoffman-Singleton graph, see for example [HaeH89]. The examination of this partition may give a more geometrical way for the proof of Proposition 11. 


\section{Computations}

In this section we are giving a short digest of various computer tools which were used by us in the course of our investigation. We do not have a goal to give a comprehensive presentation of all the computations which were done, nevertheless we hope that this digest will provide the reader with some information which may be used successfully in other situations.

\section{$7.1 \quad \mathrm{COCO}$}

COCO (COherent COnfigurations) is a computer package which was created in Moscow in 1990-1992 by I. A. Faradžev with the support of Klin. Its main features are introduced in [FarK91], most of the algorithms which were used and the general methodology are described in [FarKM94].

COCO has a number of functions which have as input two variables and as output one or two variables. The values of variables may be files with appropriate data created in advance or data introduced from the keyboard (in this case the input filename is replaced by "*"). There are two versions of COCO, the original one, which was designed to work in MS DOS on a personal computer, and the UNIX implementation by A. E. Brouwer (1992-1993). The UNIX implementation is available from the home page of Brouwer [Bro].

For our purpose we were using the following functions from COCO: ind, cgr, inm, sub, and aut. We describe below each of these functions.

- ind input1.gen input2 output1.gen output2.map

Starting from permutation group with generators in the file input1.gen this function enumerates in output2.map the elements in the orbit of the structure (e.g. $(0,\{(5,6),(6,7),(7,5)\})$, see section 5$)$ in input2 and computes (in output1.gen) the generators of the induced action according to this enumeration.

- cgr input.gen * output.cgr

constructs the 2-orbits (or colour graph) of the permutation group with generators in input.gen and lists information about the 2-orbits on the screen.

- inm input.cgr $*$ output.nrs

computes the intersection numbers of a coherent configuration (in particular case association scheme) formed by the 2-orbits in input.cgr

- sub input.nrs option output.sub

computes all mergings which are association schemes, starting from initial association scheme (coherent configuration) with intersection numbers in input.nrs. In option it can be specified that only symmetrical (s), primitive (p), or symmetrical and primitive (sp) mergings are requested. 
- aut input1.cgr input2.sub

computes the order of the automorphism group of each merging in input2.sub of the 2-orbits input1.cgr.

For the reader's convenience we present below an incomplete output listing from a computation in COCO. Some comments have been added, written in italics. These computations with COCO show that the centralizer algebra $V(\bar{H}, \Omega)$ has exactly 10 non-trivial primitive subalgebras all of which correspond to srg's.

*** system CO-CO for construction of coherent configurations ***

The file m20xs5.pos.gen contains the generators $g_{1}, g_{2}, g_{3}, g_{4}, g_{5}$ of the group $\bar{H}$. The following command constructs the induced action on the structure $\alpha$, see section 5 .

COCO> ind m20xs5.pos.gen * 100a 100a

COCO> cgr 100a *100a

This action has 13 2-orbits. The following table gives the valency of each 2-orbit and a representative $x$ so that $(0, x)$ is in that 2-orbit.

$\begin{array}{ccc}\text { number } & \text { length } & \text { representative } \\ 0 & 1 & 0 \\ 1 & 4 & 1 \\ 2 & 6 & 2 \\ 3 & 6 & 3 \\ 4 & 12 & 6 \\ 5 & 12 & 7 \\ 6 & 6 & 8 \\ 7 & 12 & 11 \\ 8 & 12 & 16 \\ 9 & 12 & 20 \\ 10 & 12 & 25 \\ 11 & 1 & 37 \\ 12 & 4 & 60\end{array}$

reflexive suborbits:0

symmetrical suborbits: $1,2,3,5,6,7,9,10,11,12$

pairs of antisymmetrical suborbits: $(4,8)$ 


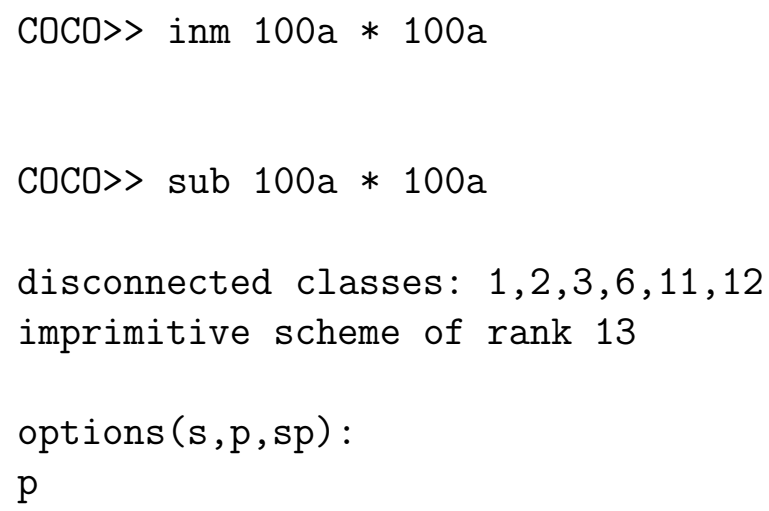

1. subscheme of rank 3 with parameters $(100,22,0)$ by merging * $(1,2,3,4,8,5,9,10,11)(6,7,12)$

2 . subscheme of rank 3 with parameters $(100,22,0)$ by merging * $(1,2,3,4,8,7,9,10,11)(5,6,12)$

3 . subscheme of rank 3 with parameters $(100,45,20)$ by merging $*$ $(1,2,3,5,9,11,12)(4,8,6,7,10)$

4. subscheme of rank 3 with parameters $(100,45,20)$ by merging $*$ $(1,2,3,5,10,11,12)(4,8,6,7,9)$

5. subscheme of rank 3 with parameters $(100,45,20)$ by merging * $(1,2,3,7,9,11,12)(4,8,5,6,10)$

6 . subscheme of rank 3 with parameters $(100,45,20)$ by merging * $(1,2,3,7,10,11,12)(4,8,5,6,9)$

7. subscheme of rank 3 with parameters $(100,36,14)$ by merging * $(1,4,8,5,6,9,11,12)(2,3,7,10)$

8. subscheme of rank 3 with parameters $(100,36,14)$ by merging * $(1,4,8,5,6,10,11,12)(2,3,7,9)$

9. subscheme of rank 3 with parameters $(100,36,14)$ by merging * $(1,4,8,6,7,9,11,12)(2,3,5,10)$

10. subscheme of rank 3 with parameters $(100,36,14)$ by merging $*$ $(1,4,8,6,7,10,11,12)(2,3,5,9)$

COCO>> aut $100 \mathrm{a} 100 \mathrm{a}$

The automorphism group of each of the above subschemes is computed.

colour graph of rank 13

transitive automorphism group of order 1200

rank=13; subdegrees: $1,4,6,6,12,12,6,12,12,12,12,1,4$

base of length 2,5 generators 


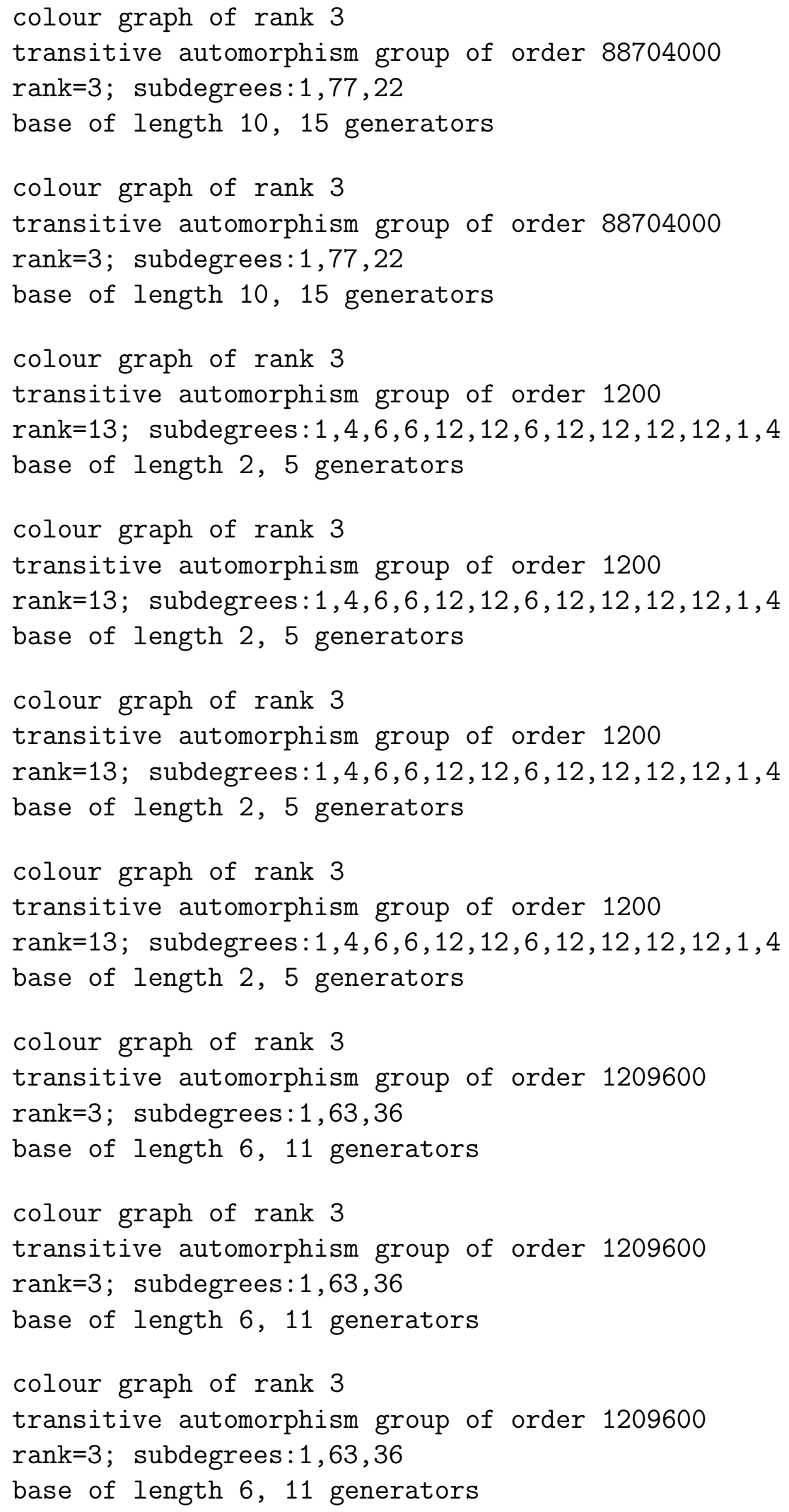




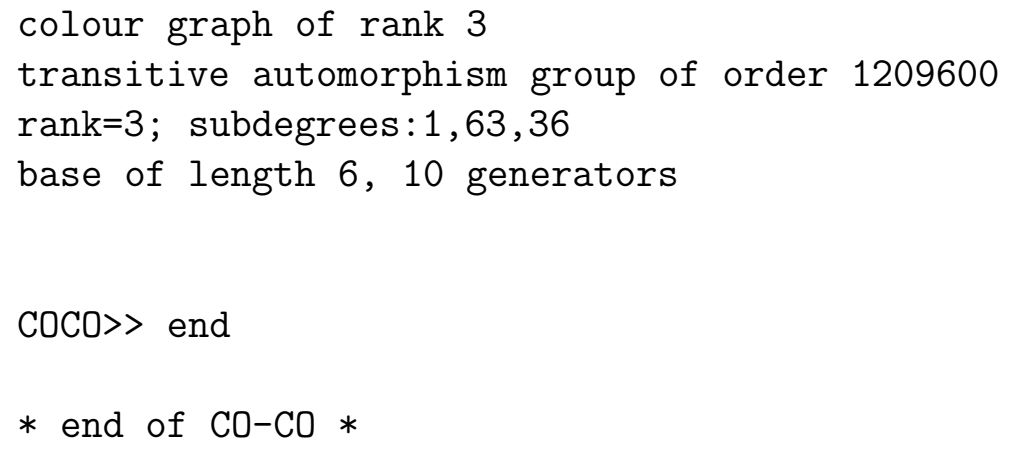

\subsection{GAP and GRAPE}

The computer package GAP (Groups, Algorithms, Programming), see [GAP] allows to arrange numerous computations in a framework of computational group theory, especially with permutation groups.

We were using GAP in various situations, for example for the fulfillment of all computational steps in the proof of Propositions 10 and 11.

The patterns of computations with GAP nowadays are available from numerous sources, including [GAP], e-messages distributed to the members of GAP-forum, scientific papers, and even books (see [Cam99]). This is why we do not include into this text any fragments from the protocols of our computations.

(Note that there exist various versions of GAP, which is continuously upgraded each 1-2 years.)

GRAPE (see [Soi93]) is a share package of GAP. An essential part of GRAPE is the program nauty by B. D. McKay [McKay90] for the computation of isomorphisms and automorphisms of graphs. GRAPE is very efficient for the investigation of a prescribed graph $\Gamma$ which is represented with the aid of a subgroup $K$ of Aut $(\Gamma)$. Such a representation in particular allows to reduce redundant routine computations in the course of the enumeration of cliques of a given size in $\Gamma$.

In spite of a difference in formats for the input and output data in COCO and GAP, the use of these two packages in join proved to be very efficient.

One of the crucial procedures, fulfilled with the aid of GAP were the computations based on the use of the shift procedure and of Proposition 9. In each of the groups $H_{3}$ and $H_{4}$ there is a subgroup $K$ of order 50 generated by $x, y$ and $w=z^{2}$ with relations $x^{5}=y^{5}=w^{2}=e, x y=y x, w x=x^{4} w, w y=y^{4} w$. For each of the regular partial difference sets $\gamma$ with parameters $(100,45,20,20)$, that we found in these groups, a new pds $\delta$ with parameters $(100,44,18,20)$ was found in the same group by applying Proposition 9 to the subgroup $K$. 


\section{Main Results}

Below we present our main "positive" results, namely discovery of a number of new pds's. A few comments show why we think that these results and their corollaries are of scientific interest. Further comments, as well as discussions of some "negative" results (i.e., of computations which do not lead to new interesting combinatorial structures), are postponed to the last Section 9 .

Proposition 12 a) There exist at least 15 regular partial difference sets over groups $H_{1}, H_{2}, H_{3}, H_{4}$ of order 100. Altogether these pds's imply 9 different (up to isomorphism) srg's.

b) There exist two srg's with parameters $(100,45,20,20)$ and $(100,44,18,20)$, respectively, both having as the automorphism group the same intransitive group $K$ of order 50 .

Remark. Two of the srg's from Proposition 12 are known. The main information about the pds's is summarised in Table 8.1. The pds's themselves are presented in Table 8.2. Here each of the pds's is presented with the aid of a string of length $k$ which includes triples $\alpha \beta \gamma$ such that $x^{\alpha} y^{\beta} z_{i}^{\gamma}$ is an element of the corresponding pds over the group $H_{i}=\left\langle x, y, z_{i}\right\rangle, i=1,2,3,4$.

The information about the graphs $\Gamma_{4}$ and $\Delta_{4}$ in part b) of Proposition 12 is also included in Table 8.1.

\begin{tabular}{|c|c|c|c|c|c|}
\hline Graph $(\Gamma)$ & pds & parameters & regular group & order of $\operatorname{Aut}(\Gamma)$ & $\operatorname{Aut}(\Gamma)$ \\
\hline$\Gamma_{1}$ & $\gamma_{1}$ & $(100,45,20,20)$ & $H_{3}$ & 1200 & $\bar{H}$ \\
\hline$\Gamma_{1}$ & $\gamma_{1}^{\prime}$ & $(100,45,20,20)$ & $H_{4}$ & 1200 & $\bar{H}$ \\
\hline$\Delta_{1}$ & $\delta_{1}$ & $(100,44,18,20)$ & $H_{3}$ & 200 & $G_{1}$ \\
\hline$\Delta_{1}$ & $\delta_{1}^{\prime}$ & $(100,44,18,20)$ & $H_{4}$ & 200 & $G_{1}$ \\
\hline$\Gamma_{2}$ & $\gamma_{2}$ & $(100,45,20,20)$ & $H_{4}$ & 100 & $H_{4}$ \\
\hline$\Delta_{2}$ & $\delta_{2}$ & $(100,44,18,20)$ & $H_{4}$ & 100 & $H_{4}$ \\
\hline$\Gamma_{3}$ & $\gamma_{3}$ & $(100,45,20,20)$ & $H_{3}$ & 100 & $H_{3}$ \\
\hline$\Delta_{3}$ & $\delta_{3}$ & $(100,44,18,20)$ & $H_{3}$ & 100 & $H_{3}$ \\
\hline$\Gamma_{4}$ & & $(100,45,20,20)$ & & 50 & $K$ \\
\hline$\Delta_{4}$ & & $(100,44,18,20)$ & & 50 & $K$ \\
\hline$\Theta$ & $\theta_{1}$ & $(100,36,14,12)$ & $H_{3}$ & $2 \cdot 604800$ & $\operatorname{Aut}\left(J_{2}\right)$ \\
\hline$\Theta$ & $\theta_{2}$ & $(100,36,14,12)$ & $H_{4}$ & $2 \cdot 604800$ & $\operatorname{Aut}\left(J_{2}\right)$ \\
\hline$\Xi$ & $\xi_{1}$ & $(100,22,0,6)$ & $H_{1}$ & $2 \cdot 44352000$ & Aut(HS) \\
\hline$\Xi$ & $\xi_{2}$ & $(100,22,0,6)$ & $H_{2}$ & $2 \cdot 44352000$ & Aut(HS) \\
\hline$\Xi$ & $\xi_{3}$ & $(100,22,0,6)$ & $H_{3}$ & $2 \cdot 44352000$ & Aut(HS) \\
\hline$\Xi$ & $\xi_{4}$ & $(100,22,0,6)$ & $H_{4}$ & $2 \cdot 44352000$ & Aut(HS) \\
\hline$\Psi$ & $\psi$ & $(100,36,14,12)$ & $H_{3}$ & 300 & $G_{2}$ \\
\hline
\end{tabular}

Table 8.1 
$\gamma_{1}$, a pds in $H_{3}$ :

010020030040120210220330340430

041111221231241321331401421441

012022032102132202222302312332402412422432442

033113143223303333343413433443

$\gamma_{1}^{\prime}$, a pds in $H_{4}$ :

010040100120130200300400420430

011031141201221231241341411431

032042102132142232242302312322342402412422442

033043123223333343403413423443

$\delta_{1}$, a pds in $H_{3}$ :

100110130140200230240300310320400410420440

041111221231241321331401421441

002042112122142212232242322342

033113143223303333343413433443

$\delta_{1}^{\prime}$, a pds in $H_{4}$ :

020030110140210220230240310320330340410440

011031141201221231241341411431

002012022112122202212222332432

033043123223333343403413423443

$\gamma_{2}$, a pds in $H_{4}$ :

110130140200210300340410420440

011111131141201211221311401421

002012022042112122132212312322342402422432442

033133223233243303313403413433

$\delta_{2}$, a pds in $H_{4}$ :

010020030040100120220230240310320330400430

011111131141201211221311401421

032102142202222232242302332412

033133223233243303313403413433

$\gamma_{3}$, a pds in $\mathrm{H}_{3}$ :

120140200210240300310340410430

201401411321031231141241341441

002012022042102132142202222242302312322332442

303403013413323133233333433143 
$\delta_{3}$, a pds in $\mathrm{H}_{3}$ :

100400010110020220320420030130230330040440

201401411321031231141241341441

402112212412122422032232432342

303403013413323133233333433143

$\theta_{1}$, a pds in $H_{3}$ :

120210220330340430

041111221231241321331401421441

012022032102132202222302312332

033113143223303333343413433443

$\theta_{2}$, a pds in $H_{4}$ :

110140200300410440

011111131141201211221311401421

012022042112122312322402432442

033133223233243303313403413433

$\xi_{1}$, a pds in $H_{1}$ :

100400

011041201421431

012042122132202312322332342402

013043103223233

$\xi_{2}$, a pds in $H_{2}$ :

100400

011041201421431

012042122132202312322332342402

013043103223233

$\xi_{3}$, a pds in $H_{3}$ :

100400

101321331411441

012042122132202312322332342402

113143203323333

$\xi_{4}$, a pds in $H_{4}$ :

100400

101321331411441

012042122132202312322332342402

113143203323333 
$\psi$, a pds in $H_{3}$ :

200300210410140340

201401411321031231141241341441

002102302312022222332042142242

303403013413323133233333433143

\section{Table 8.2}

\section{Remarks.}

1. The existence of pds $\delta_{3}$ follows implicitly from the results of K. W. Smith [Smi95] who found a reversible difference set over the group $H_{3}$ with the parameters $(100,45,20)$. Nevertheless Smith is not claiming in explicit manner the existence of the pds itself and of the corresponding srg.

2. The existence of the difference sets $\gamma_{1}, \gamma_{1}^{\prime}, \delta_{1} \cup\{e\}$ and $\delta_{1}^{\prime} \cup\{e\}$ was recently discovered independently by Golemac and Vučičić in [GolV01]. The difference sets as given in their paper are not reversible. However, reversible pds's can be found by using the shift procedure.

3. We believe that all above pds's except (implicitly) $\gamma_{1}, \gamma_{1}^{\prime}, \delta_{1}, \delta_{1}^{\prime}, \gamma_{3}$ and $\delta_{3}$ are new.

4. The four non-isomorphic srg's, $\Delta_{1}, \Delta_{2}, \Delta_{3}, \Delta_{4}$ provide existence of an srg with the parameters $(100,44,18,20)$. The problem of the existence of such srg was regarded as open in Brouwer's catalogue [Bro96] as well as by a computer daemon available from Brouwer's home page (http://www.win.tue.nl/〜aeb/).

5. The automorphism group $G_{1}$ of the graph $\Delta_{1}$ is a subgroup of $\bar{H}$. It is generated by $x, y, z \in H_{3}$ and an element $u$, such that $x^{5}=y^{5}=z^{4}=u^{2}=e, x y=y x, z x=$ $x^{2} z, z y=y^{2} z, u x=x^{4} y u, u y=y u, u z=z u$.

6. The automorphism group $G_{2}$ of the graph $\Psi$ is generated by $x, y, z \in H_{3}$ and an element $v$, such that $x^{5}=y^{5}=z^{4}=v^{3}=e, x y=y x, z x=x^{2} z, z y=y^{2} z, v x=$ $x y^{3} v, v y=x^{4} y^{3} v, v z=z v$

7. It follows from Propositions 10 and 11 that we know all pds's which correspond to the classical sporadic srg's of Hall-Wales and Higman-Sims.

Corollary 13 There exist exactly four non-isomorphic amorphic association schemes on 100 points with 3 classes having valencies 54, 36, and 9, and with the Hall-Wales graph as the relation of valency 36 .

\section{Proof}

- consider the complement $\bar{\Theta}$ of the srg of Hall-Wales, 
- find a spread $\Sigma$ in $\bar{\Theta}$, delete the edges of the spread and get a graph with the edge set $\bar{\Theta} \backslash \Sigma$,

- Then $\bar{\Theta} \backslash \Sigma, \Theta$, and $\Sigma$ provide 3 classes of a requested association scheme. There are four orbits of spreads in $\bar{\Theta}$ with representatives $\Sigma_{i}, i=1, \ldots, 4$. The graphs $\bar{\Theta} \backslash \Sigma_{i}$ are isomorphic to $\overline{\Gamma_{1}}, \overline{\Gamma_{2}}, \overline{\Gamma_{2}}$ and $\overline{\Gamma_{2}}$, and the isomorphism groups of the association scheme are the groups $\bar{H}, H_{4}, H_{4}$ and $H_{4}$, respectively.

In a similar way we can find all amorphic association schemes containing the $\operatorname{srg} \Psi$. In this case there are three orbits of spreads.

Corollary 14 There exist exactly three non-isomorphic amorphic association schemes on 100 points with 3 classes having valencies 54, 36, and 9, and with the srg $\Psi$ as the relation of valency 36 .

One of these amorphic association schemes contains the graph $\overline{\Gamma_{3}}$ as one of the relations. Another srg, $\overline{\Gamma_{4}}$ with parameters $(100,54,28,30)$ is contained as one of the relations in each of the other two amorphic association schemes. $\Gamma_{4}$ has automorphism group $K$ of order 50 with two orbits. $K$ is the index 2 subgroup of $H_{3}$ and $H_{4}$. Switching in $\Gamma_{4}$ with respect to the partition into these orbits gives a new $\operatorname{srg} \Delta_{4}$ with parameters $(100,44,18,20)$ and with the same automorphism group $K$.

Remark. According to Appendix A in [Dam99], the existence of an association scheme with the above parameters was one of the five open cases for amorphic association schemes with 3 classes having at most 100 points.

\section{Concluding remarks.}

9.1. Classical Seidel's switching is certainly the first origin of this paper. It goes back to the paper [vLinS66]. Explicitly the notion was introduced in terms of $(0,1,-1)$ adjacency matrices in [Sei67] as an equivalence relation on graphs, called complementation with respect to a subset of vertices. In this paper Seidel investigated the new operation in case of strongly regular graphs on 16 and 28 vertices. The operation was used in [BhaS68] under the name Seidel-equivalence. It seems that the term "switching" was firstly coined in $[$ BusS70].

We believe that Proposition 3 is of folklore nature. Conceptually it goes back to Seidel who was exploiting $(0,1,-1)$ matrices. Evident use of equitable partitions for similar purposes can be traced to [GodM82], where also a generalized switching was proposed and used. Our consideration of this proposition was influenced from [MuzK98].

An analogue of Seidel's switching was used in [Hae84] for the investigation of the relation between $(v, k, \lambda)$-graphs and $(v, k, \lambda)$-designs.

9.2. Main results of this paper were announced in September 2000 at Varna during the international summerschool "Algebraic Combinatorics and Computer Algebra", see 
abstract [Kli00]. Implicitly, part of our results are related to other investigations of difference sets and block designs on 100 points. The strict relations with the papers [Smi95] and [GolV01], were discussed in Section 8 (see also the recent paper [GolV02]).

A family of new difference sets with the parameters $(100,45,20)$ was described in [Vuč00]. These difference sets imply 6 non-isomorphic designs with the automorphism group $E_{25} \cdot \mathbb{Z}_{12}$ of order 300 . This group contains a regular Frobenius group of order 100 . Our computer investigation shows that no one of the difference sets discussed in [Vuč00] implies (via the shift procedure) pds's.

Another family of new difference sets with these parameters was recently found in [GolV01]. These difference sets imply 8 non-isomorphic designs. As mentioned in Section 8 , two of these designs are related to the srg's $\Gamma_{1}$ and $\Delta_{1}$. Our computer investigation shows that the remaining 6 designs are not related to any srg.

It is worth to mention that in fact the paper [Smi95], besides presentation of a new difference set, provides a very promising pattern of search for difference sets, combining initial clever restrictions approached purely theoretically with the final computer inspection of "suspicious cases".

9.3. If $\mathcal{D}=(\mathcal{P}, \mathcal{B}, \mathcal{I})$ is a symmetric $(v, k, \lambda)$-design then the dual incidence structure $\mathcal{D}^{\mathrm{T}}=\left(\mathcal{B}, \mathcal{P}, \mathcal{I}^{\mathrm{T}}\right), \mathcal{I}^{\mathrm{T}}=\{(y, x) \mid(x, y) \in \mathcal{I}\}$ is also a $(v, k, \lambda)$-design. A design $\mathcal{D}$ is called self-dual if $\mathcal{D}$ and $\mathcal{D}^{\mathrm{T}}$ are isomorphic. A duality $\sigma: \mathcal{D} \mapsto \mathcal{D}^{\mathrm{T}}$ is called a polarity if $\sigma^{2}$ acts trivially on the points and blocks of $\mathcal{D}$. A point $x \in \mathcal{P}$ of a design $\mathcal{D}$ is called an absolute point of a polarity $\sigma$ if $x \in x^{\sigma}$. Two special cases of polarities: those without absolute points, and those for which each point is absolute, are establishing special links between $(v, k, \lambda)$-designs and strongly regular graphs, see, for example, [Rud71], [CamL91] for more details.

Difference sets over abelian groups always allow polarities, see [Hal47], [BetJL93].

It turns out that the method of shifting of difference sets can be formulated in more general terms of polarities of designs. However, such consideration is not necessarily requested in the framework of our presentation.

However, as an example of practical opportunities of such more general approach, let us consider results from [JanKT01], where in particular a new $2-(100,45,20)$ design is described. The authors mention that this design is not self-dual. This automatically implies that there does not exist an srg with the parameters $(100,45,20,20)$ which corresponds to this design. To arrange a double check, we compared, using GAP, all designs discovered in our project with the design from [JanKT01] and confirmed that they are not isomorphic.

9.4. One of the initial leads in this project was to construct an srg with the parameters $(100,44,18,20)$. Our intention was to apply Proposition 3 to an srg with the parameters $(100,45,20,20)$. If in this case, $c=20$ then $a=\frac{100}{2}-2 \cdot 20=10$, and we get an $\operatorname{srg}$ with the parameters $(100,55,30,30)$, that is complementary to the desired parameters $(100,44,18,20)$.

This is why we have started from the consideration of two known examples of srg's with the parameters $(100,45,20,20)$, see [GoeS70] and [Lin83]. These graphs were examined 
with the aid of GAP. Both of these graphs have intransitive automorphism groups with one fixed vertex and the lengths of other orbits is a multiple of 9 . For both graphs, using heuristic methods, we were trying to construct an equitable partition into two equal parts. However our attempts were not successful.

Note that the parameter set $(100,45,20,20)$ is corresponding to a Latin type graph $L_{5}(10)$. Such graph may appear from a set of three mutually orthogonal Latin squares of order 10. Inspection of all known and new srg's of $L_{5}(10)$ type shows that they are pseudo$L_{5}(10)$ graphs. In our eyes the problem of the existence of three mutually orthogonal Latin squares of order 10 still remains one the most challenging tasks in "experimental" combinatorics.

9.5. One of the by-products of the use of switching is the discovery of 7 pairwise nonisomorphic 3-class amorphic association schemes with valencies 9, 36, 45 (Corollaries 13 and 14). We refer to [Dam99] for more detailed consideration of this class of association schemes. As it was mentioned earlier, the paper by van Dam contains a list of all feasible parameters for such schemes of order at most 100, together with information about the existence. At the time of preparation of our paper, for most of the parameters on 100 points the question of existence remained open. A small progress achieved in our project hopefully will stimulate further investigations of the existence of such amorphic schemes. Note that a more general question of existence of a partition of a group to $d \geq 3$ pds's is also of a definite interest.

Note that one of the remaining open cases has valencies $9,45,45$. A search for spreads $10 \circ K_{10}$ in srg's, complementary to srg's of valency 45 discovered by us, did not result in construction of such association schemes.

9.6. The strongly regular graph $\Xi$ with the parameters $(100,22,0,6)$ is known to be unique, see [Gew69]. The graphs with parameters $(100,36,14,12)$ are of $L_{4}(10)$ type. There exist a rank 3 Hall-Wales graph $\Theta$ with these parameters which is pseudo-Latin. Due to [BosPS60] there exist at least one graph of $L_{4}(10)$ type which is indeed geometrical, that is coming from a pair of orthogonal Latin squares. It was checked, using a computer, that our new $\operatorname{srg} \Psi$ is also pseudo-geometrical. An evident interesting question is to describe up to isomorphism all geometrical $L_{4}(10)$ srg's.

One more natural question is to describe all srg's with the parameters $(100,36,14,12)$ which satisfy a 4-vertex condition in the sense of [HesH71]. Each rank 3 graph, and therefore $\Theta$ has this property. We are not aware of any other example with this parameter set, satisfying the 4-vertex condition. Note that it was shown in [Wal69] that $\Theta$ can be characterised uniquely as a rank 3 graph.

9.7. We attract the reader's attention to the following phenomenon. The Higman-Sims graph $\Xi$ allows 4 partial difference sets over 4 non-Abelian groups $H_{1}, H_{2}, H_{3}, H_{4}$. All these groups have a similar representation in terms of generators $x, y, z$, see Section 6. It turns out that our strings for the pds's $\xi_{1}, \xi_{2}$ (with respect to the corresponding generators $x, y, z)$ are equal. The similar occurrence appears for the pds's $\xi_{3}, \xi_{4}$. In other words, the knowledge of one pds in each pair allows in this case to predict another pds in the same 
pair in a sense "automatically". We believe that such phenomenon can be explained theoretically by a careful inspection of the group $\bar{H}$ which is a maximal subgroup of Aut(HS) acting transitively on the vertex set of the graph $\Xi$, see [Mag71].

Note also that it will be very interesting to get a complete list of all pds's over the above-mentioned four groups.

9.8. One more interesting problem will be description of all mergings of classes of the 2-orbits of the group $G_{1}$ of order 200 in its transitive action of degree 100 on the set $\Omega$. This group is naturally described as a subgroup of $\bar{H}$, namely $G_{1} \cong\left(F_{5}^{20} \times F_{5}^{20}\right)^{\text {pos }}$ (like in Section 5). The rank of the action of $G_{1}$ on 100 points is equal to 60 , this is why we were not able find with the aid of COCO all BM-algebras of $V\left(G_{1}, \Omega\right)$.

We think that a specially arranged attack on this problem may simultaneously result in discovery of new interesting srg's, as well as imply some further progress in the development of computational tools for the investigation of coherent algebras.

(Note that the transitive group $G_{2}$ of order 300 has rank $36 . V\left(G_{2}, \Omega\right.$ ) has 24 symmetrical primitive subalgebras. All these subalgebras have rank 3 and correspond to an srg which is isomorphic to either $\Theta$ or $\Psi$. These computations were easily done with the aid of COCO.)

9.9. One more way to approach the graphs, which were discovered by us, may be based on the use of two-graphs (see, for example, [CamL91]). We intend to consider such approach in the conjunction with other methods exploited in the current paper in one of subsequent papers of this series.

9.10. Difference sets with the parameters $(100,45,20)$ play a significant role in the development of the theory of "genuinely non-abelian difference sets" (in the sense of Liebler [Lie99]). We hope that this paper will shed an extra light on the links of such objects with pds's.

9.11. When the authors had finished the preparation of this paper we became aware of a second paper by Bagchi [Bag92]. In this paper he gives a construction of the $\operatorname{srg} \Gamma_{1}$ with automorphism group of order 1200 .

Acknowledgements We thank M. Muzychuk for helpful discussions. An essential part of this project was fulfilled during visits of L. Jørgensen to Beer Sheva and M. Klin to Aalborg. We are grateful to both departments at these universities for their support and friendly attention. We thank a referee and B. McKay for helpful editorial remarks. The final version of this text was elaborated at University of Delaware, Department of Mathematical Sciences.

\section{References}

[Asc94] M. Aschbacher, Sporadic groups, Cambridge Tracts in Mathematics 104, Cambridge University Press, 1994. 
[Bab77] L. Bábái, Isomorphism problem for a class of point-symmetric structures, Acta Math. Acad. Sci. Hung. 29, 329-336, 1977.

[Bag88] B. Bagchi, A new strongly regular graph admitting the Hall-Janko-Wales group. Tech. Report No. 13/88. Stat.-Math. Division, Indian Statistical Institute, Calcutta, Sept.23, 1988.

[Bag92] B. Bagchi, A regular two-graph admitting the Hall-Janko-Wales group. Sankhya, Ser. A, Spec. Volume 54, 35-45, 1992.

[BetJL93] T. Beth, D. Jungnickel and H. Lenz, Design theory, Cambridge University Press, 1993.

[BhaS68] Bhagwandas and S. S. Shrikhande, Seidel-equivalence of strongly regular graphs, Sankhya, Ser. A 30, 359-368, 1968.

[BosPS60] R. C. Bose, E. T. Parker and S. S. Shrikhande, Further results on the construction of mutually orthogonal Latin squares and the falsity of Euler's conjecture, Canad. J. Math. 12, 189-203, 1960.

[Bro] A. E. Brouwer, Unix implementation of COCO, (http://www.win.tue.nl/〜aeb/).

[Bro96] A. E. Brouwer, Strongly regular graphs, in: The CRC Handbook of Combinatorial Designs (eds.: Colbourn and Dinitz), 667-685, CRC Press, 1996.

[BusS70] F. C. Bussemaker and J. J. Seidel, Symmetric Hadamard matrices of order 36, Annals of the New York Acad. of Sciences 175, 66-79, 1970.

[Cam99] P. J. Cameron, Permutation Groups, London Mathematical Society Student Texts 45, Cambridge University Press, 1999.

[CamL91] P. J. Cameron and J. H. van Lint, Designs, graphs, codes and their links, London Mathematical Society Student Texts 22, Cambrigde University Press, 1993.

[Dam99] E. R. van Dam, Three-class association schemes, J. Algebraic Combin. 10, 69107, 1999.

[FarK91] I. A. Faradžev and M. H. Klin, Computer package for computations with coherent configurations, Proc. of the 1991 Intern. Symp. on Symb. and Algebraic Computations, ISSAC'91 (Bonn, July 15-17, 1991), ACM Press, 219-223.

[FarKM94] I. A. Faradžev, M. H. Klin and M. E. Muzichuk, Cellular rings and groups of automorphisms of graphs, in: Investigations in algebraic theory of combinatorial objects (eds.: Faradžev, Ivanov, Klin and Woldar), Kluwer Academic Publishers, 1994, pp. 1-152.

[FinR73] L. Finkelstein and A. Rudvalis, Maximal subgroups of the Hall-Janko-Wales group, J. Algebra 24, 486-493, 1973. 
[FisM78] J. Fischer and J. McKay, The non-abelian simple groups $G,|G|<10^{6}$ - Maximal subgroups. Math. Comput. 32, 1293-1302, 1978.

[GAP] The GAP Group, GAP - Groups, Algorithms, and Programming, Version 4.1 , Aachen, St Andrews, 1999, (http://www-gap.dcs.st-and.ac.uk/ gap).

[Gew69] A. Gewirtz, Graphs with maximal even girth, Canad. J. Math. 21, 915-934, 1969.

[GodM82] C. D. Godsil and B. D. McKay, Constructing cospectral graphs, Aequationes Math. 25, 257-268, 1982.

[GoeS70] J.-M. Goethals and J.J. Seidel, Strongly regular graphs derived from combinatorial designs, Canad. J. Math. 22, 597-614, 1970.

[GolV01] A. Golemac and T. Vučičić, New difference sets in nonabelian groups of order 100, J. Combin. Designs 9, 424-434, 2001.

[GolV02] A. Golemac and T. Vučičić, New (100,45,20) symmetric designs and Bush-type Hadamard matrices of order 100, Discr. Math. 245, 263-272, 2002.

[HaeH89] W. H. Haemers and D. G. Higman, Strongly regular graphs with strongly regular decompositions, Linear Algebra Appl. 114(115), 379-398, 1989.

[Hal47] M. Hall, Cyclic projective planes, Duke Math. J. 14, 1079-1090, 1947.

[HalW68] M. Hall, Jr. and D. Wales, The simple group of order 604,800, J. Algebra 9 417-450, 1968.

[Hae84] W. Haemers, Dual Seidel switching, EUT-Rep., Eindhoven 84-WSK-03, 183-191, 1984.

[HeiK] A. Heinze and M. Klin, Constructive enumeration of partial difference sets. I. Enumeration of partial difference sets over the groups of order at most 49. In preparation.

[HesH71] M. D. Hestenes and D. G. Higman, Rank 3 groups and strongly regular graphs, SIAM-AMS Proceedings 4, 141-159, 1971.

[HigS68] D. G. Higman and C. C. Sims, A simple group of order 44,352,000, Math. Zeitschrift 105 110-113, 1968.

[HugP85] D. R. Hughes and F. C. Piper, Design Theory, Cambridge University Press, 1985.

[IvaKF82] A. A. Ivanov, M. H. Klin and I. A. Faradžev, Primitive representations of non-abelian simple groups of order less than $10^{6}$ (in Russian), Part 1, preprint, M., VNIISI, 1982. 
[IvaKF84] A. A. Ivanov, M. H. Klin and I. A. Faradžev, Primitive representations of non-abelian simple groups of order less than $10^{6}$ (in Russian), Part 2, preprint, M., VNIISI, 1984.

[Jan69] Z. Janko, Some new simple groups of finite order. I, Symposia Mathematica (INDAM, Rome, 1967/68), 1, 25-64, Academic Press, 1969.

[JanKT01] Z. Janko, H. Kharaghani and V. D. Tonchev, Bush-type Hadamard matrices and symmetric designs, J. Combin. Designs 9, 72-78, 2001.

[Kli00] M. Klin, Partial difference sets for known and new strongly regular graphs. In: Summer School ALCCAL 2000. Algebraic Combinatorics and Computer Algebra. September 3-14, 2000, Varna, Bulgaria. Inst. of Math. and Inform., Bulgarian Academy of Sciences. Sofia 2000, pp. 59-60.

[KliPR88] M. Ch. Klin, R. Pöschel and K. Rosenbaum, Angewandte Algebra für Mathematiker und Informatiker, VEB Deutscher Verlag der Wissenschaften, Berlin, 1988.

[KomT86] E. A. Komissartschik and S. V. Tsaranov, Intersections of maximal subgroups in simple groups of order less than $10^{6}$, Comm. Algebra 14, 1623-1678, 1986.

[Lie99] R.A.Liebler. Constructive representation theoretic methods and non-abelian difference sets, in: Difference sets, sequences and their correlation properties (eds: Pott, Kumar, Helleseth, Jungnickel), Kluwer Academic Publishers, 1999, pp. 331-352.

[vLinS66] J. H. van Lint and J. J. Seidel, Equilateral point sets in elliptic geometry, Indag. Math. 28, 335-348, 1966.

[Lin83] Lin Zhangwen, An example of the non-uniqueness of the $L_{5}(10)$ association scheme, Chin. Ann. of Math. 48, 355-356, 1983.

[Ma84] S. L. Ma, Partial difference sets, Discrete Math. 52, 75-89, 1984.

[Ma94] S. L. Ma, A survey of partial difference sets, Des. Codes Cryptogr. 4, 221-261, 1994.

[Mag71] S. S. Magliveras, The subgroup structure of the Higman-Sims simple group, Bull. Amer. Math. Soc. 77, 535-539, 1971.

[McKay90] B.D. McKay, nauty user's guide (version 1.5), Technical report TR-CS-90-02, Australian National University, Computer Science Department, ANU, 1990.

[MuzK98] M. Muzychuk and M. Klin, On graphs with three eigenvalues, Discrete Math. 189, 191-207, 1998.

[Rog84] N. N. Roghelia and S. S. Sane, Classification of (16,6,2)-designs by ovals, Discrete Math. 51, 161-177, 1984. 
[Rud71] A. Rudvalis, $(v, k, \lambda)$-graphs and polarities of $(v, k, \lambda)$-designs, Math. Zeitschr. 120, 224-230, 1971.

[Sei67] J. J. Seidel, Strongly regular graphs of $L_{2}$-type and of triangular type, Indag. Math. 29, 188-196, 1967.

[Soi93] L. H. Soicher. GRAPE: a system for computing with graphs and groups. In: Groups and Computation (eds.: Finkelstein and Kantor), volume 11 of DIMACS Series in Discrete Mathematics and Theoretical Computer Science, pages 287-291. American Mathematical Society, 1993.

[Smi95] K. W. Smith, Non-abelian Hadamard difference sets, J. Combin. Theory Ser. A 70, 144-156, 1995.

[Tur65] R. J. Turyn, Character sums and difference sets, Pacific J. Math. 15, 319-346, 1965.

[Vuč00] T. Vučičić, New symmetric designs and nonabelian difference sets with parameters $(100,45,20)$, J. Combin. Designs 8, 291-299, 2000.

[Wal69] D. Wales, Uniqueness of the graph of a rank three group, Pacific J. Math. 30, 271-276, 1969.

[Wil85] R. A. Wilson, Maximal subgroups of automorphism groups of simple groups, $J$. London Math. Soc. (2) 32, 460-466, 1985. 